\title{
shinyBN: an online application for interactive Bayesian network inference and visualization
}

\author{
Jiajin Chen ${ }^{1}$, Ruyang Zhang ${ }^{1}$, Xuesi Dong ${ }^{2}$, Lijuan Lin', Ying Zhu', Jieyu He ${ }^{1}$, David C. Christiani ${ }^{3}$ \\ Yongyue Wei ${ }^{i^{*}}$ and Feng Chen ${ }^{1,4^{*}}$
}

\begin{abstract}
Background: High-throughput technologies have brought tremendous changes to biological domains, and the resulting high-dimensional data has also posed enormous challenges to computational science. A Bayesian network is a probabilistic graphical model represented by a directed acyclic graph, which provides concise semantics to describe the relationship between entities and has an independence assumption that is suitable for sparse omics data. Bayesian networks have been broadly used in biomedical research fields, including disease risk assessment and prognostic prediction. However, the inference and visualization of Bayesian networks are unfriendly to the users lacking programming skills.

Results: We developed an R/Shiny application, shinyBN, which is an online graphical user interface to facilitate the inference and visualization of Bayesian networks. shinyBN supports multiple types of input and provides flexible settings for network rendering and inference. For output, users can download network plots, prediction results and external validation results in publication-ready high-resolution figures.

Conclusion: Our user-friendly application (shinyBN) provides users with an easy method for Bayesian network modeling, inference and visualization via mouse clicks. shinyBN can be used in the R environment or online and is compatible with three major operating systems, including Windows, Linux and Mac OS. shinyBN is deployed at https://jiajin.shinyapps.io/shinyBN/. Source codes and the manual are freely available at https://github.com/ JiajinChen/shinyBN.
\end{abstract}

Keywords: Bayesian network, Visualization, Inference, Online tool, R package

\section{Background}

Bayesian networks have become one of the most commonly used models for the modeling and reasoning of uncertain systems. In the biomedical field, Bayesian networks are successfully applied to assess the risk of disease and explore the relationship between genotypes and phenotypes [1, 2]. However, the inference and visualization of Bayesian networks is not user friendly. SMILE (Structural Modeling, Inference, and Learning Engine) is a causal discovery engine [3] and is easily embedded into the other tools, such as jSMILE, a Java

\footnotetext{
* Correspondence: ywei@njmu.edu.cn; fengchen@njmu.edu.cn ${ }^{1}$ Department of Biostatistics, School of Public Health, State Key Laboratory of Reproductive Medicine, Nanjing Medical University, Nanjing, China Full list of author information is available at the end of the article
}

implementation of SMILE, and rSMILE, an R package connecting to jSMILE [4]. However, since SMILE has been shifted from open license to commercial version (product brand: GeNIe), rSMILE and jSMILE are no longer maintained. BayesianNetwork, an R/Shiny web widget to construct Bayesian network [5], while the connections between nodes are nondirectional, and only one predictor variable can be considered for outcome inference which hinders its application in real-word medical studies. In addition, there are some commercial products for Bayesian network analysis which require complex installation (Table 1). To solve these inconveniences, we developed shinyBN, an online tool based on $\mathrm{R}$ and Shiny for interactive inference and visualization of Bayesian network, incorporating multiple types of inputs, 
Table 1 Existing tools for Bayesian network analysis

\begin{tabular}{llllllll}
\hline Tool & Latest version & Free & Open source & Web based & R API & Interactive visualization & Batch inference \\
\hline jSMILE/ rSMILE & $2009^{a}$ & Y & N & N & Y & Y & Y \\
Netica & 2018 & $N^{b}$ & N & N & N & Y & N \\
BayesiaLab & 2018 & N & N & N & N & Y & Y \\
BayesianNetwork & 2018 & Y & Y & Y & Y & N & N \\
GeNle/ SMILE & 2019 & $N^{c}$ & N & N & Y & Y & Y \\
Bayes Server & 2019 & N & N & N & Y & Y & Y \\
shinyBN & 2019 & Y & Y & Y & Y & Y &
\end{tabular}

aStop maintenance

${ }^{\mathrm{b}}$ Maximal 15 nodes allowed for free version

${ }^{\mathrm{c}}$ Free only for academic community

flexible parameter settings, and multiple combinations of outcomes.

\section{Implementation}

\section{Overview of shinyBN}

shiny $B N$ was developed with five $\mathrm{R}$ packages:

- bnlearn for structure learning and parameter training [6];

- gRain for network inference [7];

- visNetwork for network visualization [8];

- $p R O C$ for plotting receiver operating characteristic (ROC) curves [9];

- rmda for plotting the decision curve analysis (DCA); and was further wrapped by R/Shiny, a framework to build interactive web applications by $\mathrm{R}$ [10]. By using these packages, shinyBN could construct the Bayesian network by the uploaded structural information from Excel file or R object, learning the Bayesian network by individual data, visualize and customize the network illustration, and implement the network for outcome inference. A flow chart of the proposed shiny $B N$ is shown in Fig. 1. shiny $B N$ is compatible with three major operating systems and popular browsers (Additional file 2).

\section{Network input}

shinyBN supports three types of input:

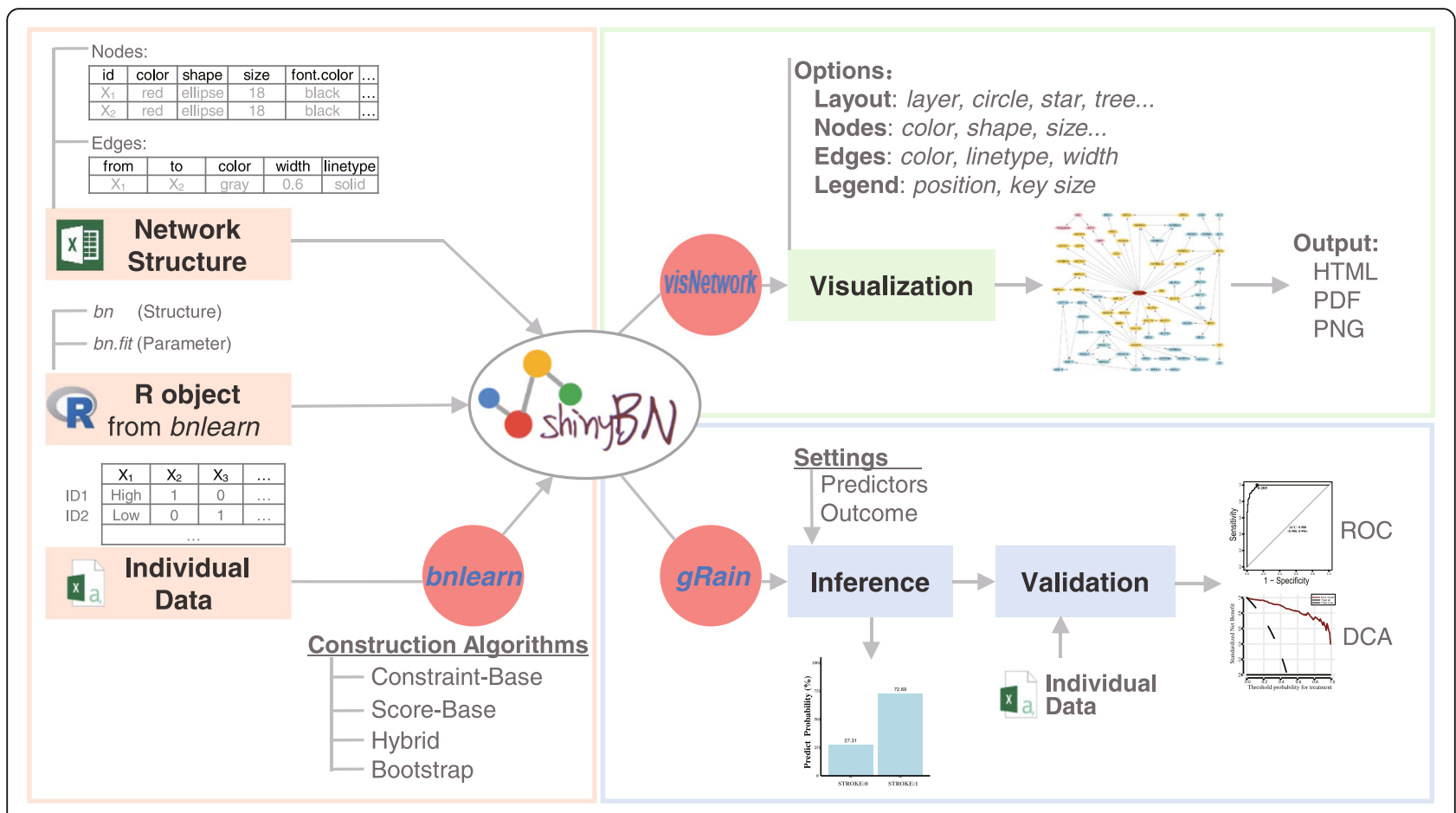

Fig. 1 The flow chart of the proposed shinyBN application 
- Microsoft Excel file, which has network structural information and properties of the nodes (size, color, shape) and edges (color, width and line type);

- bnlearn output object that embeds Bayesian network (class bn or bn.fit);

- csv file with individual data for Bayesian network structure learning and parameter training. The data is an $\mathrm{N} \times \mathrm{M}$ matrix with discrete data, where $\mathrm{N}$ is the number of observables and $\mathrm{M}$ is the number of the features (nodes).

\section{Network construction}

Bayesian network constructions are performed using the methods in the bnlearn $\mathrm{R}$ package [6]. Users can select constraint-based algorithms, score-based algorithms or hybrid algorithms to train the network structure and incorporate structural priors by setting whitelists (included in the graph) and blacklists (excluded from the graph), and the bootstrap approach is supported in shinyBN as well [11]. Parameter estimation via either maximum likelihood estimation or the Bayesian method is supported in shinyBN. The structure information (nodes, edges) of constructed network can be further extracted for visualization using visNetwork. The network can be directly transformed to class grain that met the requirement of the input for gRain package and perform inference.

\section{Network visualization}

Network visualizations are based on the visNetwork $\mathrm{R}$ package using vis.js JavaScript library [8]. Once the input is uploaded to the server, a visualization of the network with default settings is automatically rendered. The properties of the nodes and edges can be modified by changing the corresponding settings. Node color can be defined individually, by color palettes that meet scientific journal requirements, or by the dominant colors automatically extracted from the uploaded picture. The widths of the connections can be defined manually or corresponding to the strength of the probabilistic relationships. For a better presentation, graph layouts can be modified by the default layouts or, conveniently, by mouse drag and drop. A high-resolution network graph can be downloaded from shinyBN.

\section{Outcome inference}

Inferences are performed using the junction tree algorithm in gRain R package [7]. It transforms a Bayesian network model into a tree, combines the efficiency of belief propagation and the sum-product method to allow the efficient computation of posterior probabilities.

By selecting the nodes of interest as outcomes, defining the factors (nodes) as predictive variables, setting the values accordingly as evidence, the predicted results will be displayed in a bar plot or a probabilistic table. Marginal and joint prediction results for multiple outcomes can be output. In addition, shinyBN supports external validation sets uploaded for batch inference and outputs the inference results, an ROC curve, a DCA curve and other evaluation indices. Publication-ready high-resolution figures can be downloaded from shinyBN.

\section{Timing evaluation}

The performance of the application largely depends on the configurations of the computer. In order to improve the performance, the shiny server is upgraded with $8 \mathrm{~GB}$ of RAM. We evaluated the timing of shinyBN by using several publicly accessible networks with different number of nodes (Table 2).

\section{Results}

\section{Real data application}

Stroke is a severe complication of sickle cell anemia (SCA) that can cause permanent brain damage and even death. By integrating 108 SNPs from 39 candidate genes and clinical characteristics from 1398 individuals with SCA, Sebastiani et al. constructed a Bayesian network to predict the risk of stroke, which achieved an excellent accuracy of $98.2 \%$ [1].

First, the network model was replicated using the information from the original study and uploaded to shi$n y B N$. Size and color of nodes and width of edges could be modified for a better presentation. In the example, the color of the nodes for the clinical characteristics was set to pink, and the color of the Markov blanket for stroke, which directly associated with stroke, was set to yellow. The layout of network was manually adjusted by the mouse drag and drop (Fig. 2). By setting the evidence for some candidate gene loci, the predicted

Table 2 Timing evaluation of shiny $B N$

\begin{tabular}{lllll}
\hline Network & Number of nodes & Number of edges & Time for visualization & Time for single inference \\
\hline Cancer & 5 & 4 & $1 \mathrm{~s}$ & $2 \mathrm{~s}$ \\
Child & 20 & 25 & $1 \mathrm{~s}$ & $2 \mathrm{~s}$ \\
Hailfinder & 56 & 66 & $1 \mathrm{~s}$ & $2 \mathrm{~s}$ \\
Pathfinder & 109 & 195 & $1 \mathrm{~s}$ & $2 \mathrm{~s}$ \\
Diabetes & 413 & 602 & $2 \mathrm{~s}$ & $7 \mathrm{~s}$ \\
Munin (full network) & 1041 & 1397 & $2 \mathrm{~s}$ & $12 \mathrm{~s}$ \\
\hline
\end{tabular}




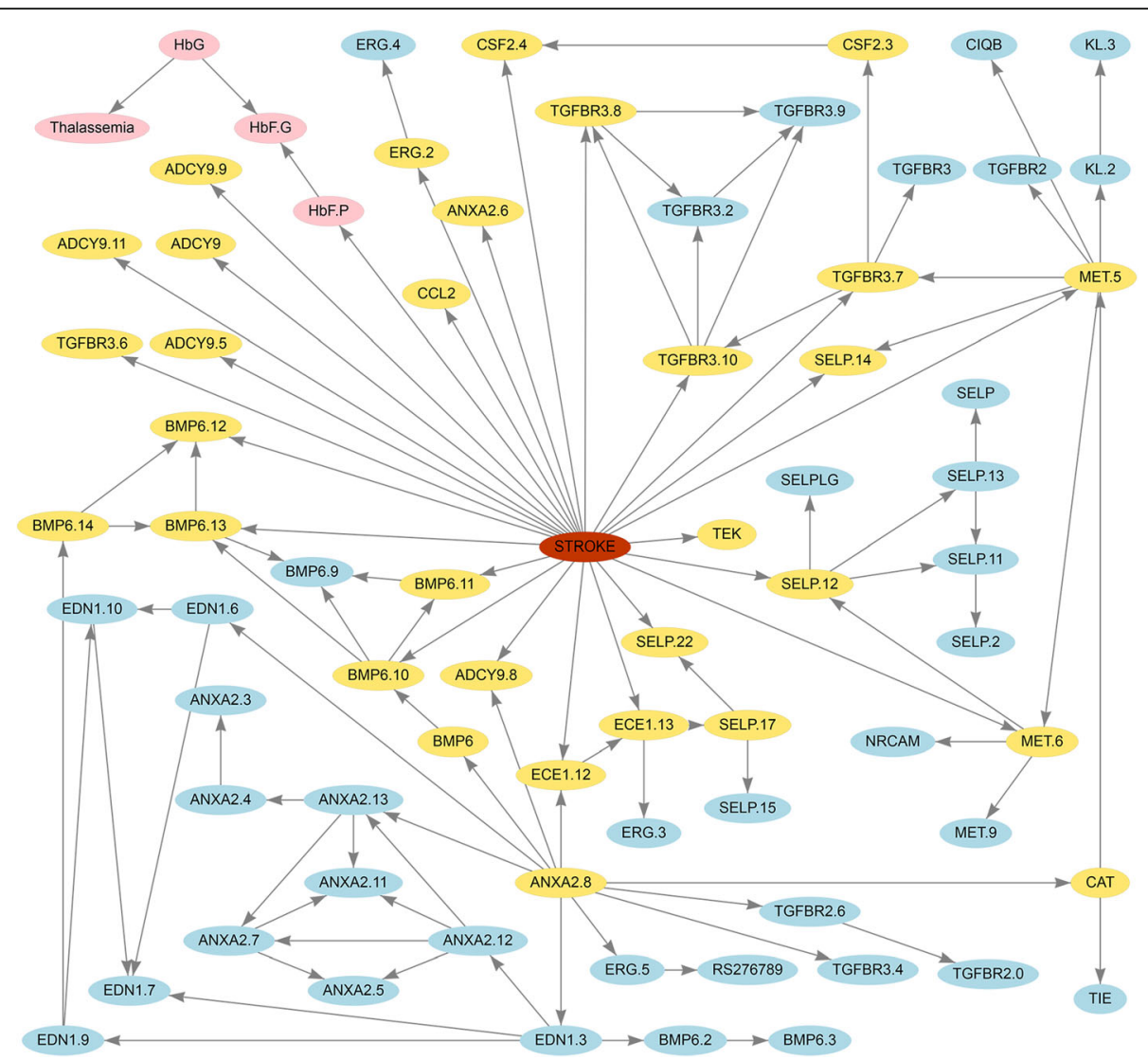

Fig. 2 The stroke network rendered by shinyBN

probabilities for stroke are displayed in a table and a bar plot (Additional file 3). The network can be downloaded as an HTML file for high-resolution figures or in as an Excel file for network structures. Furthermore, we simulated random data from the stroke Bayesian network with a missing rate of $40 \%$ for each variable and then uploaded it to the server as a pseudo external validation set. The ROC plot (Additional file 4) and the DCA plot (Additional file 5) were displayed, and the batch inference results were download as a comma-separated values file.

\section{Conclusions}

In conclusion, we developed an online application, shi$n y B N$, to construct and illustrate a Bayesian network with high scalability. shinyBN supports multiple types of input and provides flexible settings for network rendering and inference. A real data application confirms that the Bayesian network can be used for omics data modeling. By integrating several packages, shiny $B N$ is a practical pipeline for Bayesian network modeling, inference and visualization.

\section{Availability and requirements}

Project name: shinyBN.

Project home page: https://github.com/JiajinChen/ shinyBN

Operating system(s):

For $\mathrm{R}$ users, any platform for which the $\mathrm{R}$ software is implemented;

For online users, any platform with compatible browser.

Programming language: $R$

Other requirements: Shiny

License: Apache License 2.0

Any restrictions to use by non-academics: None

\section{Supplementary information}

Supplementary information accompanies this paper at https://doi.org/10. 1186/s12859-019-3309-0.

Additional file 1. A zip archive containing the source codes and manual of shinyBN.

Additional file 2. The compatibility of the proposed shinyBN application. We tested the compatibility of shinyBN across three major operating systems and popular browsers.

Additional file 3. The inference result generated by shinyBN. (A) The settings of the evidence for some candidate gene loci; (B) The predicted 
probability of stroke displayed in a probabilistic table; (C) The predicted probability of stroke displayed in a bar plot.

Additional file 4. The ROC plot for the simulated validation set. This file contains the receiver operating characteristic curve for the simulated validation set of the stroke network.

Additional file 5. The DCA plot for the simulated validation set. This file contains the decision curve for the simulated validation set of the stroke network.

Additional file 6. An R script containing all the code to construct the stroke network using conditional probability tables provided in [1].

Additional file 7. A zip archive containing the testing files of shinyBN.

\section{Abbreviations}

API: Application programming interface; DCA: Decision curve analysis; HTML: HyperText Markup Language; ROC: Receiver operating characteristic; SCA: Sickle cell anemia; SMILE: Structural modeling, inference, and learning engine; SNP: Single nucleotide polymorphism

\section{Acknowledgements}

The authors thank the contributors of the cited $\mathrm{R}$ packages.

\section{Authors' contributions}

$J C$ wrote the codes and the manuscript. YW and FC conceived the idea and designed the software. DCC revised the manuscript critically. RZ, XD and LL optimized the code. $\mathrm{YZ}$ and $\mathrm{JH}$ extensively tested the application. All authors read and approved the final manuscript.

\section{Funding}

This work has been supported by the National Key Research and Development Program of China (2016YFE0204900 to F.C.), the National Natural Science Foundation of China (81530088 to F.C., 81973142 to Y.W.), the US National Institutes of Health (CA209414, CA092824, and ES000002 to D.C.C.). The funding sponsors had no role in study design, collection, analysis and interpretation of data, or preparation of the manuscript.

\section{Availability of data and materials}

The proposed shinyBN is deployed at https://jiajin.shinyapps.io/shinyBN/. Source codes and manual are freely available at https://github.com/ JiajinChen/shinyBN and Additional file 1. The R scripts to construct the stroke network are included as Additional file 6. The testing files for users are included as Additional file 7.

\section{Ethics approval and consent to participate}

Not applicable.

\section{Consent for publication}

Not applicable.

\section{Competing interests}

The authors declare that they have no competing interests.

\section{Author details}

'Department of Biostatistics, School of Public Health, State Key Laboratory of Reproductive Medicine, Nanjing Medical University, Nanjing, China. ${ }^{2}$ Department of Epidemiology and Biostatistics, School of Public Health, Southeast University, Nanjing, China. ${ }^{3}$ Department of Environmental Health, Harvard School of Public Health, Boston, MA, USA. ${ }^{4}$ Jiangsu Key Lab of Cancer Biomarkers, Prevention and Treatment, Jiangsu Collaborative Innovation Center for Cancer Personalized Medicine, Nanjing Medical University, Nanjing, China.

Received: 26 March 2019 Accepted: 5 December 2019

Published online: 16 December 2019

\section{References}

1. Sebastiani P, Ramoni MF, Nolan V, Baldwin CT, Steinberg MH. Genetic dissection and prognostic modeling of overt stroke in sickle cell anemia. Nat Genet. 2005;37(4):435.
2. Krishnan KC, Kurt Z, Barrere-Cain R, Sabir S, Das A, Floyd R, Vergnes L, Zhao $Y$, Che N, Charugundla S. Integration of multi-omics data from mouse diversity panel highlights mitochondrial dysfunction in non-alcoholic fatty liver disease. Cell Syst. 2018;6(1):103-115.e107.

3. Druzdzel MJ. SMILE: structural modeling, inference, and learning engine and GeNle: a development environment for graphical decision-theoretic models. In: Sixteenth National Conference on Artificial Intelligence and Eleventh Conference on Innovative Applications of Artificial Intelligence; 1999. p. 902-3.

4. Friedrich CM, Klinger R. rSMILE, an interface to the Bayesian Network package GeNle/SMILE. In: Book of Abstracts of the R User Conference; 2009.

5. Govan PB. BayesianNetwork: interactive Bayesian network modeling and analysis. J Open Source Softw. 2018;3:425.

6. Scutari M. Learning Bayesian networks with the bnlearn R package. J Stat Softw. 2010:35(03):2010.

7. Højsgaard S. Graphical independence networks with the gRain package for R. J Stat Softw. 2012;46(10):1-26.

8. Almende B, Thieurmel B, Robert T. visNetwork: network visualization using'vis. js' Library. R Package Version 2.0.9. 2019; https://CRAN.R-project. org/package=visNetwork.

9. Robin X, Turck N, Hainard A, Tiberti N, Lisacek F, Sanchez J-C, Müller M. pROC: an open-source package for $\mathrm{R}$ and $\mathrm{S}+$ to analyze and compare ROC curves. BMC Bioinformatics. 2011;12(1):77.

10. Chang W, Cheng J, Allaire JJ, Xie Y, Mcpherson J. shiny: web application framework for R. R Package Version 1.4.0. 2019; https://CRAN.R-project.org/ package $=$ shiny.

11. Friedman N, Goldszmidt M, Wyner A. Data analysis with Bayesian networks: a bootstrap approach. In: Proceedings of the Fifteenth Conference on Uncertainty in Artificial Intelligence. San Francisco: Morgan Kaufmann Publishers Inc; 1999. p. 196-205.

\section{Publisher's Note}

Springer Nature remains neutral with regard to jurisdictional claims in published maps and institutional affiliations.

\section{Ready to submit your research? Choose BMC and benefit from:}

- fast, convenient online submission

- thorough peer review by experienced researchers in your field

- rapid publication on acceptance

- support for research data, including large and complex data types

- gold Open Access which fosters wider collaboration and increased citations

- maximum visibility for your research: over $100 \mathrm{M}$ website views per year

At BMC, research is always in progress.

Learn more biomedcentral.com/submissions 